Revista Destaques Acadêmicos, Lajeado, v. 8, n. 3, 2016. ISSN 2176-3070 DOI: http://dx.doi.org/10.22410/issn.2176-3070.v8i3a2016.1150 www.univates.br/revistas

\title{
PRESENÇA DE POLÔNIO-210 NO TABACO: UMA REVISÃO SISTEMÁTICA
}

\author{
Daniel Nuncio ${ }^{1}$, Fernanda Rocha da Trindade ${ }^{2}$
}

Resumo: Este trabalho teve por finalidade revisar os estudos na literatura envolvendo o Polônio-210 $\left({ }^{210} \mathrm{Po}\right)$ de forma geral e relacionado com o tabagismo. A pesquisa ocorreu em artigos científicos publicados no portal BIREME e suas respectivas bases de dados (MEDLINE, LILACS, DECS, HISA e IBECS), somando 944 publicações internacionais envolvendo o ${ }^{210} \mathrm{Po}$. Destas referências, 239 artigos foram selecionados por possuírem texto completo disponível, sendo utilizados 53 efetivamente. Considerados os estudos já realizados, torna-se necessária a realização de novas pesquisas laboratoriais e populacionais envolvendo a presença do Polônio-210 no tabaco. Mesmo que comprovada esta presença, não houve uma padronização referente ao método de pesquisa e detecção deste elemento químico radioativo.

Palavras-chave: Polônio-210. Tabagismo. Câncer.

\section{INTRODUÇÃO}

O alemão Wilhelm Conrad Roentgen anunciou em 8 de novembro de 1895 a descoberta dos raios catódicos, aos quais denominou de raios X. Este foi o primeiro indício comprovado da existência da radiação ionizante. No ano seguinte, Antoine Henry Becquerel, Pierre e Marie Curie realizaram experiências com Urânio e comprovaram a existência da radioatividade (MARTINS, 1990). O Polônio-210 ( $\left.{ }^{210} \mathrm{Po}\right)$ é um elemento químico radioativo com meia-vida igual a 138,4 dias, que emite radiação ionizante a partir da geração de partícula alfa e radiação gama (HUNT; RUMNEY, 2007).

A partir de diversos trabalhos científicos (TSO et al., 1966; AOUN, 2010; SEILER; WIELMES, 2012, entre outros), sabe-se da existência do ${ }^{210} \mathrm{Po}$ em plantas e nas folhas do tabaco (MUGGLI et al., 2008). Comprovadamente, existe

1 Tecnólogo em Radiologia, Acadêmico de Biomedicina da Univates. E-mail: drnuncio@hotmail.com

2 Física Médica, Mestre em Ciências Médicas; Docente da Univates. E-mail: fernanda.trindade@univates.br 
um risco à saúde dos seres vivos gerado pela exposição ao ${ }^{210} \mathrm{Po}$ (SCOTT, 2007; LI, 2007; WARROUX et al., 2011) através da sua presença no tabaco (TSO et al., 1966; HOLTZMAN; ILCEWICZ, 1966; KHATER, 2004; AOUN, 2010; SEILER; WIELMES 2012; BOICE et al., 2014).

As embalagens de cigarros conhecidas no comércio atual, assim como dados de estudos científicos, informam que há mais de 4.000 substâncias compostas no cigarro e a emissão de 0,3 a 3,3 bilhões de partículas emitidas por mililitro de fumaça de cigarro (ZAGÀ et al., 2011). Mesmo com esta informação, não há menção de quais são estas partículas ou substâncias e a quantidade estimada para cada unidade do produto. Para a população em geral, que não é usuária de tabaco, o risco de exposição ao ${ }^{210} \mathrm{Po}$ é, teoricamente, inexistente, pois para que ocorram danos é necessário haver contaminação direta por ingestão ou inalação e não exposição à fumaça na forma passiva (KELECOM et al., 1998; SCOTT, 2007; LI, 2007). A partir dessas considerações, este estudo teve o objetivo de analisar a associação do ${ }^{210}$ Po com o tabaco, a partir de publicações científicas ao longo dos anos.

\section{MATERIAIS E MÉTODOS}

O estudo foi realizado entre os meses de fevereiro e julho de 2016. Neste período, realizou-se uma pesquisa de revisão sistemática em artigos científicos publicados no portal BIREME, nas bases LILACS (Literatura LatinoAmericana e do Caribe em Saúde), MEDLINE (Medical Literature Analysis and Retrieval System Online), DECS (Descritores em Ciências da Saúde), HISA (Base Bibliográfica em História da Saúde Pública na América Latina e Caribe) e IBECS (Índice Bibliográfico Espanhol em Ciências da Saúde) referente ao ${ }^{210} \mathrm{Po}$, contextualizando-o na agricultura, tabaco, intoxicações humanas e métodos para a sua detecção desenvolvidos até o momento.

Para tanto, buscou-se artigos a partir do termo "Polônio" em língua portuguesa, sendo que automaticamente a ferramenta de busca do site trouxe dados nacionais e internacionais sobre o tema, obtendo artigos em 14 idiomas. Como critério de seleção, optou-se por artigos de texto completo. Os artigos com conteúdo completo disponíveis para consulta, se reduziram a 4 idiomas. Apenas um artigo foi encontrado em língua portuguesa (MARTINS, 1990).

A partir do termo descritor, foram encontrados artigos sobre o tema nas bases de dados MEDLINE (936 artigos), LILACS (4 artigos), DECS (2 artigos), HISA ( 1 artigo) e IBECS (1 artigo), somando no total 944 publicações. Entre todos os radioisótopos do elemento químico Polônio, foi considerado para este estudo, apenas $\mathrm{O}{ }^{210} \mathrm{Po}$, pelo fato de este ser o componente acumulado nas folhas de tabaco (TSO et al., 1966; HOLTZMAN; ILCEWICZ, 1966; KHATER, 2004; MUGGLI et al., 2008; AOUN, 2010; SEILER; WIELMES 2012; BOICE et al., 2014). 
Do total de 944 publicações, 239 eram artigos com texto completo disponível. Destes, obteve-se um número de 150 artigos com pertinência sobre o tema. Em análise final, destacaram-se 52 artigos relatando de forma objetiva a ligação de ${ }^{210} \mathrm{Po}$ e tabaco, sendo então utilizados de base efetiva para este estudo.

Os artigos foram separados em quatro temas referentes ao ${ }^{210} \mathrm{Po}$ : a) Relação entre elementos radioativos encontrados no solo, ar, água e alimentos; b) Acidentes envolvendo o elemento químico ${ }^{210} \mathrm{Po}$; c) Métodos de detecção desenvolvidos; d) Relação entre patologias humanas, tabaco e ${ }^{210} \mathrm{Po}$.

\section{RESULTADOS E DISCUSSÃO}

O Polônio foi descoberto pelo casal Pierre e Marie Curie, em uma comunicação à Academia de Ciências, em 12 de abril de 1898 (CURIE, 1899; MARTINS, 1990; LE, 2007). O casal anunciou que a pechblenda, um minério contendo óxido de urânio, era bem mais radioativo (cerca de 400 vezes mais) que o urânio metálico isolado. Isso significava que o minério continha, além do urânio, outro elemento radioativo (MARTINS, 1990).

O polônio possui 41 isótopos, com massas variando de 187 a 227 (ANSOBORLO, 2014). Segundo Hunt \& Rumney (2007), o esquema básico de decaimento natural simplificado até chegar no ${ }^{210} \mathrm{Po}$, se dá na seguinte ordem: Chumbo-210 $\left({ }^{210} \mathrm{~Pb}\right.$, decaimento $\beta, \mathrm{t}^{1 / 2} 22,3$ anos $) \rightarrow$ Bismuto- $210\left({ }^{210} \mathrm{Bi}\right.$, decaimento $\beta, \mathrm{t}^{1} \frac{2}{2} 5,0$ dias) $\rightarrow{ }^{210} \mathrm{Po}$ (decaimento $\alpha$ e gama, $\mathrm{t}^{1} \frac{1}{2} 138,4$ dias). Este esquema básico é gerado a partir da série que se inicia no elemento primário deste decaimento, dado pelos respectivos radioisótopos: Urânio-238 $\left({ }^{238} \mathrm{U}\right) \rightarrow$ Tório-234 $\left({ }^{234} \mathrm{Th}\right) \rightarrow$ Protactínio-234 ${ }^{\left({ }^{234} \mathrm{~Pa}\right)} \rightarrow$ Urânio-234 $\left({ }^{234} \mathrm{U}\right) \rightarrow$ Tório-230 $\left({ }^{230} \mathrm{Th}\right) \rightarrow$ Rádio-226 $\left({ }^{226} \mathrm{Ra}\right) \rightarrow$ Radônio-222 $\left({ }^{222} \mathrm{Rn}\right)$.

$\mathrm{O}{ }^{210} \mathrm{Po}$ possui alta energia transferida através da partícula alfa (KELECOM et al., 1998), com energia de emissão na ordem de 5,3 MeV, emitindo também fótons gama com energia de $803 \mathrm{keV}$ (ZAGÀ et al., 2011). As partículas alfa são facilmente barradas pela roupa, folha de papel ou queratina da pele, porém ao entrar em contato com células vivas são vinte vezes mais prejudiciais, comparado com raios $\mathrm{X}$ e gama. Sua toxicidade se dá ao ser ingerido, inalado ou absorvido através de alguma lesão na pele (LE, 2007). Comparando com outros elementos radioativos, $1 \mathrm{mg}$ de ${ }^{210}$ Po pode emitir tantas partículas alfa quanto $5 g$ de ${ }^{226} \mathrm{Ra}$ (ZAGÀ et al., 2011).

\section{Relação entre elementos radioativos encontrados no solo, ar, água e alimentos}

Pesquisadores acrescentam o interesse radio-ecológico do ${ }^{210} \mathrm{Po}$, por conter mais de $90 \%$ da dose de exposição natural de diversas espécies naturais (ARUNACHALAM et al., 2014), pois é encontrado no solo e atmosfera, com níveis de concentração mais elevados no fumo do tabaco (LE, 2007, 
ANSOBORLO, 2014). O solo é uma das principais fontes de avaliação para um marcador de distribuição e migração da série de radionuclídeos do Urânio. $\mathrm{O}$ aumento da umidade no solo, reduz a taxa e difusão do gás ${ }^{222} \mathrm{Rn}$, portanto, condições climáticas estão diretamente envolvidas com os depósitos de metais pesados responsáveis pelo surgimento do ${ }^{210} \mathrm{Po}$. As regiões mais secas e de pouca umidade são locais com uma provável concentração maior deste radioisótopo no solo, pela maior facilidade de dispersão no ar através das partículas de Aitken (PERSSON, 2009; ARUCHANALAM et al., 2014).

Em uma pesquisa italiana, mais de 120 alimentos de consumo humano foram identificados contendo concentrações de ${ }^{210} \mathrm{Po}$ em sua composição, sendo $67 \%$ desta fonte advinda de frutos do mar, especialmente mariscos (MELI et al., 2014), porém, grandes consumidores destes alimentos não apresentaram elevação na quantidade média de concentração de ${ }^{210} \mathrm{Po}$ no organismo (HUNT \& RUMNEY, 2007). Anchovas e sardinhas tem bioacumulação da concentração ao longo da cadeia alimentar (STRADY et al., 2015).

De acordo com Matthews e colaboradores (2007), o ${ }^{210} \mathrm{Po}$ é analisado para uma variedade de fins, incluindo para a avaliação de impacto radiológico, como marcador de processos ambientais e como uma medida indireta de seu gerador, ${ }^{210} \mathrm{~Pb}$. Seus usos específicos englobam o estudo de efeitos de partículas alfa e calibração de detectores de radiação, além de ser uma fonte concentrada de calor (ANSOBORLO, 2014).

Relatos de estudos realizados nos Estados Unidos apontam a presença de ${ }^{210}$ Po na água potável e em produtos alimentares de animais criados em áreas contaminadas. $O$ estudo nestas regiões indicou um acúmulo do elemento radioativo em ovários, destruindo oócitos primários, acúmulo em saco vitelino do embrião, tecidos fetais e placentários, gerando efeitos com exposição de baixo nível a longo prazo (SEILER; WIELMES, 2012).

\section{Acidentes envolvendo o elemento químico ${ }^{210} \mathrm{Po}$}

Le (2007) descreve Irene Joliot-Curie, filha de Marie e Pierre Curie como a primeira pessoa conhecida que teve morte por exposição após uma explosão acidental de selados de ${ }^{210} \mathrm{Po}$, falecendo em Paris 10 anos depois, por leucemia. Contudo, Ansoborlo (2014) aponta um acidente no laboratório dos Curie logo depois da sua descoberta, acusando a morte de um técnico após liberação acidental do radioisótopo em um vaso de destilação.

Mais recentemente, em 23 de novembro de 2006, a morte de um ex espião russo (Alexander Litvinenko) foi associada ao envenenamento por ${ }^{210} \mathrm{Po}$ em Londres e atraiu a atenção do público para a cinética, dosimetria e o risco da toxicidade deste radioisótopo ao ser humano (SCOTT, 2007; LI, 2007; LI et al., 2010, ANSOBORLO, 2014), assim como as detecções do radioisótopo nos profissionais de saúde que cuidaram dele no período de internação (WAROUX et al., 2011). Este episódio, em termos de segurança interna, gerou um alerta 
laranja (alto risco de atentados), sendo o ${ }^{210}$ Po classificado como arma terrorista em potencial e arma radiológica (LE, 2007).

Houve suspeitas de que o líder israelense Yasser Arafat também tenha sido assassinado por envenenamento da mesma forma que o ex-espião russo (ANSOBORLO, 2014), mas nada foi comprovado por evidências médicas diretas, o que foi tratado como mera especulação (FESSEL, 2013; NADEL, 2013). A quantidade necessária para veneno deve ser fabricada artificialmente, bombardeando bismuto natural $\left({ }^{209} \mathrm{Bi}\right)$ com nêutrons ou partículas alfa com energia de $37 \mathrm{MeV}$, sendo ambos processos altamente burocráticos e de acesso controlado, realizado em reatores de usinas nucleares (LE, 2007; ANSOBORLO, 2014).

A ingestão de ${ }^{210} \mathrm{Po}$ e consequente emissão de partículas alfa para os seres humanos, possui uma variabilidade de fatores que podem levar à dose letal, variando nos tecidos em um parâmetro determinado pela fração da dose e a eficácia biológica relativa (RBE), comparada com raios $x$ e raios gama (HARRISON et al., 2007). Ele é considerado 10.000 vezes mais tóxico do que o Cianeto de Hidrogênio, e juntamente com a toxina botulínica, uma das substâncias tóxicas conhecidas (ANSOBORLO, 2014). A Internacional Agency for Research on Cancer (IARC) considera o ${ }^{210} \mathrm{Po}$ como um carcinógeno humano do Grupo 1, descrito quando há evidências suficientes de que o agente é carcinogênico para humanos (SEILER; WIELMES, 2012). Na ausência de tratamento médico, a dose letal em humanos por contaminação via oral é da ordem de 10 a 30 microgramas (JEFFERSON et al., 2009). Há oxidação tetravalente bastante estável em água, com tendência de hidrólise e formação de coloides de $\mathrm{Po}(\mathrm{OH})$ (ANSOBORLO, 2014).

A dose letal média (DL 50) para uma exposição à radiação aguda é de cerca de $4 \mathrm{~Sv}$, correspondendo ao equivalente a ingestão de $50 \mathrm{ng}$ ou inalação de $10 \mathrm{ng}$ de ${ }^{210} \mathrm{Po}$, com afinidade de concentração nos seres humanos em fígado, medula óssea, sistema gastrointestinal e SNC (LE, 2007; ANSOBORLO, 2014).

\section{Métodos de detecção desenvolvidos}

Para a determinação de ${ }^{210} \mathrm{Po}$ em amostras ambientais, são utilizados vários métodos, incluindo a deposição espontânea em placas de prata ou níquel, medidas por espectrometria de partículas alfa, usando um semicondutor ou $\mathrm{ZnS}(\mathrm{Ag})$ detector de partículas alfa, cromatografia, técnicas de eletrodeposição, extração em cocktail de cintilação, membranas de contenção metálica e troca iônica (JOKELAINEN et al., 2010; DLUGOSZ-LISIECKA; WRÓBEL, 2014). É necessário extrair previamente o Urânio da amostra, para que este não aja como interferente (JOKELAINEN et al., 2010). A espectroscopia de partículas alfa foi o método de exame mais encontrado para a determinação de amostras em fezes, urina e cabelo (RÄÄF et al., 2015). Não há um padrão para a determinação laboratorial deste radioisótopo, portanto a detecção pode ser realizada de várias 
maneiras experimentais, alguns com precisão de 95\% (MATTHEWS et al., 2007; LIN; WU, 2009, PLANINSEK et al., 2013).

A Tabela 1 apresenta a lista de artigos sobre o conhecimento de radioatividade e radiação ionizante, os acidentes envolvendo o elemento químico ${ }^{210}$ Po e os métodos de detecção desenvolvidos.

Tabela 1: Artigos sobre o conhecimento de radioatividade e radiação ionizante, os acidentes envolvendo o elemento químico ${ }^{210} \mathrm{Po}$ e os métodos de detecção já desenvolvidos

\begin{tabular}{|c|c|c|c|c|}
\hline $\begin{array}{l}\text { Variáveis } \\
\text { Envolvidas }\end{array}$ & Autores & Ano & $\begin{array}{l}\text { Local da } \\
\text { Pesquisa }\end{array}$ & $\begin{array}{c}\text { Síntese Temática } \\
\text { (ligação com a variável) } \\
\end{array}$ \\
\hline \multirow{8}{*}{$\begin{array}{l}\text { Acidentes } \\
\text { envolvendo } \\
\text { o elemento } \\
\text { químico }{ }^{210} \text { Po }\end{array}$} & Ansoborlo, Eric & 2014 & França & Envenenamento por ${ }^{210} \mathrm{Po}$ \\
\hline & Boice et al. & 2014 & USA & Envenenamento por ${ }^{210} \mathrm{Po}$ \\
\hline & Harrison et al. & 2007 & Inglaterra & Envenenamento por ${ }^{210} \mathrm{Po}$ \\
\hline & Jefferson et al. & 2009 & USA & Envenenamento por ${ }^{210} \mathrm{Po}$ \\
\hline & Le, Mai H. & 2007 & USA & Envenenamento por ${ }^{210} \mathrm{Po}$ \\
\hline & Scott, Bobby R. & 2007 & USA & Envenenamento por ${ }^{210} \mathrm{Po}$ \\
\hline & The Lancet & 2013 & Inglaterra & Envenenamento por ${ }^{210} \mathrm{Po}$ \\
\hline & Waroux et al. & 2011 & Inglaterra & Envenenamento por ${ }^{210} \mathrm{Po}$ \\
\hline \multirow{23}{*}{$\begin{array}{c}\text { Métodos de } \\
\text { detecção já } \\
\text { desenvolvidos }\end{array}$} & Ansoborlo, Eric & 2014 & França & Separação radioquímica \\
\hline & Aoun et al. & 2010 & $\begin{array}{c}\text { Costa } \\
\text { Mediterrânea } \\
\end{array}$ & Espectrometria alfa \\
\hline & Aoun et al. & 2015 & Líbano & $\begin{array}{c}\text { Placas de prata/ } \\
\text { Espectrometria alfa }\end{array}$ \\
\hline & Arunachalam et al. & 2014 & Índia & Deposição placas de prata \\
\hline & Begy et al. & 2013 & Romênia & Deposição espontânea \\
\hline & Boice et al. & 2014 & USA & Extração em urina \\
\hline & Bretthauer; Black & 1967 & USA & Filtros de resina \\
\hline & Curie, Marie & 1899 & França & Extração radioquímica \\
\hline & $\begin{array}{l}\text { Długosz-Lisiecka; } \\
\text { Wróbel }\end{array}$ & 2014 & Polônia & $\begin{array}{c}\text { Placas de prata/ } \\
\text { Espectrometria alfa }\end{array}$ \\
\hline & Długosz-Lisiecka & 2015 & Polônia & Espectrometria Alfa \\
\hline & Ferri; Baratta & 1966 & USA & $\begin{array}{c}\text { Placas de prata/ } \\
\text { Espectrometria alfa }\end{array}$ \\
\hline & Guérin;Dai & 2013 & Canadá & Espectrometria alfa \\
\hline & Guérin; Dai & 2015 & Canadá & $\begin{array}{l}\text { Sulfeto de Cobre/ } \\
\text { Espectrometria alfa }\end{array}$ \\
\hline & Hunt; Rumney & 2007 & Inglaterra & Espectrometria alfa \\
\hline & Jokelainen et al. & 2010 & Finlândia & Extração por solventes \\
\hline & Kather, Ashraf E.M. & 2004 & Egito & Espectrometria alfa \\
\hline & Li et al. & 2010 & Canadá & Espectrometria alfa \\
\hline & Li et al. & 2007 & Alemanha & Deposição/disco de cobre \\
\hline & Lin; $\mathrm{Wu}$ & 2009 & USA & Espectrometria alfa \\
\hline & Matthews et al. & 2007 & Nova Zelândia & Espectrometria alfa \\
\hline & Planinsek et al. & 2013 & Eslovênia & Extração em cabelo \\
\hline & Rääf et al. & 2015 & Suécia & Espectrometria alfa \\
\hline & Radford; Martell & 1975 & USA & Deposição/discos de prata \\
\hline
\end{tabular}




\section{Relação entre as patologias humanas, o tabaco e o ${ }^{210} \mathrm{Po}$}

A ligação do ${ }^{210} \mathrm{Po}$ com o câncer de pulmão iniciou a partir das experiências de Winder \& Graham, em 1950 e Holtzman no ano de 1967, entre outras (RADFORD; HUNT, 1964; HOLTZMAN, 1967). Tidd (2008) associou causas e apontou os riscos da presença do Radônio e seus produtos radioativos descendentes, sendo um destes o ${ }^{210} \mathrm{Po}$, surgido do decaimento radioativo do urânio, que é ubíquo no solo.

$\mathrm{O}{ }^{238} \mathrm{U}$ tem uma meia-vida muito longa (4,5 bilhões de anos) e vai acumular-se no solo com repetidas aplicações de fertilizante. Como resultado, os cigarros modernos podem conter níveis mais elevados de ${ }^{210} \mathrm{Po}$ comparativamente às medições de 40 anos atrás (TIDD, 2008), pelo aumento da absorção de fosfatos de cálcio. Tso e colaboradores (1966) e Ansoborlo (2014) explicam a presença de ${ }^{210} \mathrm{Po}$ à fosfatos contendo ${ }^{210} \mathrm{~Pb}$ absorvidos através da raiz da planta de tabaco, acumulando-se em suas folhas, a partir da decomposição de fertilizantes de fosfato de cálcio contaminados com urânio usados nestas plantações de tabaco. Arunachalam e colaboradores (2014) apresentaram dados que descrevem o gás ${ }^{222} \mathrm{Rn}$ como fonte primária de ${ }^{210} \mathrm{Po}$, sendo este produto de seu decaimento, bem como sua concentração no solo, ambientes aquáticos e plantas sendo feita através da deposição seca ou por aerossóis. Segundo Zagà e colaboradores (2011), neste processo de absorção pela raiz do tabaco, há esta concentração de radioisótopos na composição da planta, resultante, em segunda análise, de eventos meteorológicos como ventos, chuva e neve. Os acúmulos são triconômicos (poros filamentosos que facilitam o acúmulo de metais pesados), descritos e testados, onde há a menção ao ${ }^{222} \mathrm{Rd}$ absorvido pela poeira atmosférica (partículas de Aitken) como agente formador de ${ }^{210} \mathrm{~Pb} \mathrm{e}^{210} \mathrm{Po}$ através de decaimento encontra-se no artigo de ZAGÀ et al. (2011).

A contaminação, se dá através de partículas sub-microscópicas de ${ }^{210} \mathrm{Po}$ no ar que estão presas em pelos pegajosos sobre as folhas de plantas de tabaco. Estes pelos são bastante hidrofóbicos e, uma vez aderidos, a radioatividade não é lavada na chuva, mantendo assim a sua presença efetiva até o momento da combustão, no ato de fumar (RADFORD; MARTELL, 1975). Outras culturas não são afetadas (TIDD, 2008).

Devido ao reconhecimento da carcinogenicidade de baixos níveis de radônio em fumantes, parece possível que os baixos níveis de ${ }^{210} \mathrm{Po}$ em cigarros sejam responsáveis por alguns tipos de câncer de pulmão (TSO et al., 1966; TIDD, 2008).

Um dos indicadores da presença deste radioisótopo no ar atmosférico, também responsável pela formação de aerossóis e partículas livres de ${ }^{210} \mathrm{Po}$, é a queima do carvão nas casas ou em escala industrial. Porém, de modo geral, excetuando-se locais de poluição atmosférica, formadores das partículas de Aitken, a qualidade do ar em relação à presença de radioisótopos, está dentro dos padrões aceitáveis por todas as referências de estudos realizados. (YAN 

DLUGOSZ-LISIECKA, 2015).

Em humanos, o ${ }^{210}$ Po possui excreção fecal na proporção de 50 a $90 \%$ de seu total (LE, 2007; ZAGÀ et al., 2011), sendo o restante distribuído pelo corpo com concentrações mais elevadas no plasma, baço, rins e fígado. Seus maiores depósitos são inicialmente encontrados na corrente sanguínea, onde as células endoteliais vasculares são alvo primário para os efeitos de curto prazo. Sua radiação também afeta tecidos de reservas de células-tronco e em nível celular, causam efeitos mutagênicos e citotóxicos, devido à quebra simples ou dupla da fita de DNA (LE, 2007).

Em um estudo envolvendo a concentração em 5 órgãos do corpo humano (pulmão, fígado, rim, coração e músculo psoas) somente não foi registrada mudança significativa de acúmulo no músculo psoas (FERRI; BARATTA, 1966).

Fisiologicamente, o ${ }^{210} \mathrm{Po}$ foi testado em ratos, havendo registros de concentrações em fígado e rins em quantidades variadas (LI et al., 2010), estando seu metabolismo em humanos ainda sendo estudado de forma indireta, fato explicado pela alta toxicidade e risco de morte (SCOTT, 2007; LI, 2007; LI et al., 2010; WAROUX et al., 2011).

Um estudo com teor ocupacional considerou o número de 7.270 colaboradores que trabalharam em uma usina nuclear em Ohio (EUA) de 1944 a 1972, onde o ${ }^{210}$ Po e Berílio foram usados como fontes de nêutrons para armas nucleares. Duzentas mil amostras de urina foram analisadas para estimar as doses de radiação para órgãos do corpo, tanto de Polônio, como de outros radionuclídeos depositados internamente. $\mathrm{O}$ polônio foi o maior contribuinte para a dose de pulmão por inalação, com um risco relativo para câncer de pulmão em doses com aferições de $100 \mathrm{mSv}$ (BOICE et al., 2014).

De maneira geral, a relação entre o ${ }^{210} \mathrm{Po}$ e o tabaco diverge em datas de publicação do primeiro estudo. Rego (2009) descreve como sendo a primeira publicação sobre o assunto datada de 1964, havendo nas décadas seguintes outros trabalhos ligando radioisótopos e tabagismo.

Relatos de Bogden e colaboradores (1981) fazem uma análise de tabacos de países com incidências altas e baixas de câncer de pulmão e mostraram que as concentrações de ${ }^{210} \mathrm{Po}$ foram semelhantes, assim como os teores de alcatrão e nicotina na fumaça de cigarro. Pode-se analisar desta maneira que as concentrações de ${ }^{210} \mathrm{Po}$ estão distribuídas de forma presente e uniforme no cigarro, mas não pode ser apontada como a única causa para este tipo de neoplasia.

Para os não-tabagistas, a maior parte da ingesta natural diária de ${ }^{210} \mathrm{Po}$ $(77,3 \%)$ vem dos alimentos; $4,7 \%$ provém da água e $0,6 \%$ está contido no ar. Para os tabagistas, a inalação de fumaça do cigarro é responsável por $17,4 \%$ do total de ${ }^{210} \mathrm{Po} /$ dia. A maioria (99\%) da radioatividade do cigarro, provém do ${ }^{210} \mathrm{Po}$, e o restante se distribui entre Tório $(\mathrm{Th})$, Rádio $(\mathrm{Ra}) \mathrm{e}^{210} \mathrm{~Pb}$, tendo afinidade pela 
bifurcação brônquica, onde fica retido por ação muco-ciliar diminuída nesta região específica (ZAGÀ et al., 2011).

O provável dano tóxico é aumentado, pois esta quantidade é muito maior do que os 5,3\% ingeridos/inalados através da água e do ar respirado no dia, levando a acreditar que os altos níveis de radiotoxicidade e energia das partículas alfa causarão efeitos a curto, médio ou longo prazo no organismo do tabagista, de forma bastante elevada.

Concentrações de ${ }^{210} \mathrm{Po}$ e ${ }^{210} \mathrm{~Pb}$ foram encontradas em quantidade dobrada nos pulmões de fumantes, em relação aos não-fumantes (HOLTZMAN; ILCEWICZ, 1966). Khater (2004) testou diversos componentes do cigarro através do método de espectrometria alfa, usando detectores de barreira de superfície, após a separação química do ${ }^{210} \mathrm{Po}$.

Os indivíduos tabagistas, ao consumir um maço de cigarro (20 unidades) estão inalando em média $123 \mathrm{mBq}$ por dia de ${ }^{210} \mathrm{Po}$ e ${ }^{210} \mathrm{~Pb}$ (doses individuais estimadas para cada um). Os valores médios de dose efetiva anual para fumantes (um maço por dia) foram estimadas em 193 e $251 \mu \mathrm{Sv}$ de ${ }^{210} \mathrm{Po}$ e ${ }^{210} \mathrm{~Pb}$, respectivamente (KHATER, 2004). Dados estes valores, fazendo-se uma correlação com a DL-50 para humanos, estimada em $1100 \mathrm{~Bq}$, o que equivalente

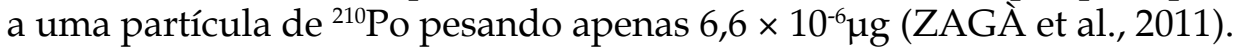

Considerando o consumo de dois maços de cigarros por dia, temse em um ano, uma dose de radiação estimada de $0,4 \mathrm{~Sv}$ a $10 \mathrm{~Sv}$ em 25 anos (RADFORD; HUNT, 1964), fazendo com que, anualmente, o tabagista esteja exposto a uma taxa de dose de radiação cerca de 150 vezes maior do que a dose natural para este mesmo período, estimada em cerca de 0,05 Sv (ZAGÀ et al., 2011).

Bretthauer e Black (1967) sugeriam a utilização de uma resina de troca iônica mista como um filtro para cigarros, na intenção de minimizar os efeitos do ${ }^{210} \mathrm{Po}$ para os tabagistas. A lavagem ácida foi descoberta em 1980 para ser altamente eficaz na remoção de ${ }^{210} \mathrm{Po}$ a partir das folhas do tabaco porém, assim como a alternativa anterior, não é aplicada porque a indústria tabagista considera prejudicial tal procedimento por ocasionar perda da chamada "nicotina kick", uma má absorção de nicotina no cérebro dos fumantes (KARAGUEUZIAN et al., 2011).

Um estudo propôs que o tabagismo pode ser uma grande fonte de ingestão diária de ${ }^{210} \mathrm{Po}$ e ${ }^{210} \mathrm{~Pb}$, após testes realizados também pelo método de espectrometria alfa. Na Itália, foram estudadas 10 marcas de cigarros do comércio local encontrando-se associação do ${ }^{210} \mathrm{Po}$ com o cigarro (TARONI et al., 2014), assim como na Romênia, em estudo semelhante (BEGY et al., 2013).

Em outro panorama comparou-se cigarros indianos, fabricados a partir de plantas cultivadas em terrenos com baixo uso de fertilizantes, e outro cultivado com uso de fertilizantes (cigarros americanos). $\mathrm{O}$ resultado para os primeiros foi um valor de 6 a 15 vezes menos radioativos, e em termos 
gerais de quantificação os cigarros brasileiros possuem maior concentração de radioisótopos comparados aos cigarros gregos e indianos (ZAGÀ et al., 2011).

Para tentar reduzir a quantidade de ${ }^{210}$ Po no tabaco, Zaga e colaboradores (2011) sugeriram medidas como o uso de polifosfatos alternativos (adubo orgânico), evitar o armazenamento por longo período (aumento da concentração nas folhas), colhendo as folhas ainda verdes, a manipulação genética das folhas de tabaco, diminuindo a quantidade de tricomas e por conseguinte, a redução do acúmulo dos radioisótopos, filtros de resinas, uso de bactérias específicas para diminuir biologicamente a concentração de polifosfatos nas rochas, e sinalização da presença do ${ }^{210}$ Po nas embalagens de cigarros.

$\mathrm{Na}$ Tabela 2 estão os artigos que apresentaram a relação existente entre os elementos radioativos encontrados no solo, ar, água e alimentos e entre as patologias humanas, o tabaco e o ${ }^{210} \mathrm{Po}$.

Tabela 2: Lista com os artigos que apresentaram: a) a relação que existe entre elementos radioativos encontrados no solo, ar, água e alimentos; e b) relação entre patologias humanas, tabaco ${ }^{210} \mathrm{Po}$

\begin{tabular}{|c|c|c|c|c|}
\hline $\begin{array}{l}\text { Variáveis } \\
\text { Envolvidas }\end{array}$ & Autores & Ano & $\begin{array}{l}\text { Local da } \\
\text { Pesquisa }\end{array}$ & $\begin{array}{l}\text { Síntese Temática } \\
\text { (ligação com a } \\
\text { variável) }\end{array}$ \\
\hline \multirow{12}{*}{$\begin{array}{l}\text { Relação que } \\
\text { existe entre } \\
\text { elementos } \\
\text { radioativos } \\
\text { encontrados } \\
\text { no solo, } \\
\text { ar, água e } \\
\text { alimentos }\end{array}$} & Aoun et al. & 2010 & $\begin{array}{c}\text { Costa } \\
\text { Mediterrânea }\end{array}$ & Solo \\
\hline & Curie, Marie & 1899 & França & Minério de Urânio \\
\hline & $\begin{array}{c}\text { Długosz-Lisiecka \& } \\
\text { Wróbel }\end{array}$ & 2014 & Polônia & Ar, musgo e líquen \\
\hline & Dtugosz-Lisiecka & 2015 & Polônia & $\begin{array}{c}{ }^{210} \text { Po no ar } \\
\text { atmosférico }\end{array}$ \\
\hline & Hunt \& Rumney & 2007 & Inglaterra & Marisco e caranguejo \\
\hline & Jokelainen et al. & 2010 & Finlândia & $\begin{array}{c}{ }^{234} \mathrm{U},{ }^{238} \mathrm{U},{ }^{210} \text { Po na } \\
\text { água }\end{array}$ \\
\hline & Kelecom et al. & 1999 & Brasil & Plantas aquáticas \\
\hline & Meli et al. & 2014 & Itália & $\begin{array}{c}\text { Legumes, vegetais, } \\
\text { frutas } \\
\end{array}$ \\
\hline & Persson \& Holm & 2014 & $\begin{array}{c}\text { Oceano Ártico/ } \\
\text { Antártico }\end{array}$ & ${ }^{210} \mathrm{~Pb},{ }^{7} \mathrm{Be},{ }^{210} \mathrm{Po}$ no ar \\
\hline & Strady et al. & 2015 & França & $\begin{array}{c}\text { Água do mar e } \\
\text { Anchovas }\end{array}$ \\
\hline & Tso et al. & 1966 & USA & Solo \\
\hline & Yan et al. & 2012 & Coréia do Sul & $\begin{array}{c}\text { Ar atmosférico e } \\
\text { chuva }\end{array}$ \\
\hline
\end{tabular}




\begin{tabular}{|c|c|c|c|c|}
\hline $\begin{array}{l}\text { Variáveis } \\
\text { Envolvidas }\end{array}$ & Autores & Ano & $\begin{array}{l}\text { Local da } \\
\text { Pesquisa }\end{array}$ & $\begin{array}{l}\text { Síntese Temática } \\
\text { (ligação com a } \\
\text { variável) }\end{array}$ \\
\hline \multirow{20}{*}{$\begin{array}{l}\text { Relação entre } \\
\text { patologias } \\
\text { humanas, } \\
\text { tabaco e e }{ }^{210} \text { Po }\end{array}$} & Adler, Isaac & 1912 & USA & Câncer de pulmão \\
\hline & Begy et al. & 2013 & Romênia & Câncer de pulmão \\
\hline & Boice et al. & 2014 & USA & $\begin{array}{l}\text { Câncer de esôfago, } \\
\text { pulmão e leucemia }\end{array}$ \\
\hline & Bogden et al. & 1981 & USA & Câncer de pulmão \\
\hline & Bretthauer \& Black & 1967 & USA & Câncer de pulmão \\
\hline & Ferri \& Baratta & 1966 & USA & Câncer de pulmão \\
\hline & Holtzman, Richard B. & 1967 & USA & Câncer de pulmão \\
\hline & Holtzman \& Ilcewicz & 1966 & USA & Câncer de pulmão \\
\hline & $\underline{\text { Karagueuzian et al. }}$ & 2011 & USA & Câncer de pulmão \\
\hline & Kather, Ashraf E.M. & 2004 & Egito & Câncer de pulmão \\
\hline & Marshall, T.R. & 2014 & USA & $\begin{array}{l}\text { Câncer de pulmão e } \\
\text { doenças cardíacas }\end{array}$ \\
\hline & Muggli et al. & 2008 & USA & Câncer e mutações \\
\hline & Rego, Bryanna & 2009 & USA & Câncer de pulmão \\
\hline & Radford \& Hunt & 1964 & USA & Câncer de pulmão \\
\hline & Radford \& Martell & 1975 & USA & Câncer de pulmão \\
\hline & Seiler \& Wiemels & 2012 & USA & Leucemia e mutações \\
\hline & Taroni et al. & 2014 & Itália & Bronquite e Câncer \\
\hline & Tidd, Michael D. & 2008 & USA & Câncer de pulmão \\
\hline & Winder \& Graham & 1950 & USA & Câncer broncogênico \\
\hline & Zága et al. & 2011 & Itália & Câncer de pulmão \\
\hline
\end{tabular}

\section{CONCLUSÃO}

Considerados os estudos já realizados, torna-se necessária a realização de novas pesquisas laboratoriais e populacionais envolvendo a presença do ${ }^{210}$ Po no tabaco visando, entre outros, a padronização da metodologia de estudo para tipificar cada amostra em questão, além de uma descrição mínima da quantidade das substâncias mais tóxicas contidas no cigarro (incluindo o ${ }^{210} \mathrm{Po}$ ).

Sugere-se listar tais substâncias em quantidade por unidade do produto, de forma ampla, à exemplo do que já existe nas embalagens de alimentos de consumo humano e animal encontrados no comércio. Além disto, novos estudos também são desejáveis para esclarecer com precisão o mecanismo de interação deste radioisótopo com células animais de diferentes tecidos.

\section{REFERÊNCIAS}

ADLER, Isaac A. Primary Malignant Growths of the Lung. CA-A Cancer Journal for Clinicians. Vol.30 n.5: 295-301, September/October, 1980. 
ANSOBORLO, Eric. Poisonous polonium. Nature Chemistry, Vol. 6: May, 2014.

AOUN, M; EL SAMRANI, A. G; LARTIGES, B.S; KAZPARD, V; SAAD, Z. Releases of Phosphate Fertilizer Industry in the Surrounding Environment: Investigation on Heavy Metals and Polonium-210 in Soil. Journal of Enviromental Sciences (China); Vol. 22(9): 1387-1397, 2010.

AOUN, M; EL SAMAD, O; KHOZAM, B. R; LOBINSKI, R. Assessment of committed effective dose due to the ingestion of $210 \mathrm{Po}$ and $210 \mathrm{~Pb}$ in consumed Lebanese fish affected by a phosphate fertilizer plant. Journal of Environmental Radioactivity. 140: 25-29, 2015.

ARUNACHALAM, Kantha D; BASKARAM, Kamesh V; RAO, D. D; SATHYAPRIYA, R;ANNAMALAI, Sathesh K; KURUVA, JAYA K; HARI, S. Ingestion of Polonium (210Po) via dietary sources in high background radiation areas of south India. International Journal of Radiation Biology; Early Online: 1-9, 2014.

BEGY, R. C; SOMLAI, J; KOVACS, T; DUMITRU (RUSU), O. A; COSMA, C. The Activity concentration of 210-Po in romanian comercial cigarretes and the radiation exposure estimation derived from their regular consumption. Radiation Protection Dosimetry (2013), pp. 1-5, may, 2013.

BOICE, John D. Jr; COHEN, Sarah S; MUMMA, Michael T; ELLIS, Elizabeth Dupree; CRAGLE, Donna L; ECKERMAN, Keith F; WALLACE, Phillip W; CHADDA, Bandana; SONDERMAN, Jennifer S; WIGGS, Laurie D; RICHTER, Bonnie S; LEGGET, Richard W. Mortality Among Mound Workers Exposed to Polonium-210 and Other Sources of Radiation, 1944-1979. Radiation Research 181(2):208-228, february, 2014.

BOGDEN, John. D; KEMP, Francis. W; BUSE, Marga; THIND, Inderjit. S; LOURIA, Donald. B; FORGACS, Joseph; LLANOS, Guillermo; TERRONES, Ignacio Moncoya. Composition of tobaccos from countries with high and low incidences of lung cancer. I. Selenium, polonium-210, alternarla, tar, and nicotine. Journal of the National Cancer Institute, 66: 27-31, 1981.

BRETTHAUER, Erich W; BLACK, Stuart C. Polonium-210: removal from smoke by resin filters. Science 9; 156(3780):1375-6, June, 1967.

CURIE, Marie Sklodowska. Les rayons de Becquerel et le polonium. Révue Générale des Sciences, 10, 41-50, 1899.

DLUGOSZ-LISIECKA, Magdalena; WRÓBEL, Justyna. Use of moss and lichen species to identify210Po-contaminated regions. Environmental Science Processes \& Impacts. 16: 16, 2729-2733, 2014.

DLUGOSZ-LISIECKA, Magdalena. Excess of 210polonium activity in the surface urban atmosphere. Part (1) fluctuation of the 210Po excess in the air. Environmental Science Processes \& Impacts. 17: 458-467, 2015. 
FESSEL, Jeffrey. Correspondence: Political Lancet. The Lancet. Vol. 382: 1980, 14 december, 2013.

FERRI, Esther S; BARATTA, Edmond J. Polonium 210 in Tobacco, Cigarette Smoke and Selected Human Organs. Public Health Reports. Vol. 81, No. 2,121-127: February, 1966.

GUÉRIN, Nicolas; DAI, Xiongxin. Rapid Preparation of Polonium Counting Sources for Alpha Spectrometry Using Copper Sulfide Microprecipitation. Analytical Chemistry; June, 2013.

GUÉRIN, Nicolas; DAI, Xiongxin. An emergency bioassay method for ${ }^{210}$ Po in urine. Applied Radiation and Isotopes. Volume 103, Pages 179-184, Sep, 2015.

HARRISON, John; LEGGET, Rich; LLOYD, David; PHIPPS, Alan; SCOTT, Bobby. Polonium-210 as a poison. Journal of Radiological Protection; 27: 17-40, 2007.

HOLTZMAN, Richard B. Polonium-210 in bronchial epithelium of cigarette smokers. Science (New York, N.Y.), 155(3762), 607, 1967.

HOLTZMAN, Richard B; ILCEWICZ, Frank H. Lead-210 and polonium-210 in tissues of cigarette smokers. Science, 153(3741): 1259-60, sep. 1966.

HUNT, G.J; RUMNEY, H.S. The human alimentary tract transfer and body retention of environmental polonium-210. Journal of Radiological Protection; 27: 405-426, 2007.

JEFFERSON, R. D; GOANS, R. E; BLAIN, P.G; THOMAS, S. H. Diagnosis and treatment of polonium poisoning. Clinical Toxicology (Phila), 47(6):608, Jul, 2009.

JOKELAINEN, L; VESTERBACKA, P; LEHTO, J. Method validation in solvent extraction for 210Po determination from groundwaters. Radiochim; Acta 98, 91-97, 2010.

KARAGUEUZIAN, Hrayr S; WHITE, Celia; SAYRE, James; NORMAN, Amos. Cigarette Smoke Radioactivity and Lung Cancer Risk. Nicotine \& Tobacco Research 14(1):79-90, Sep. 2011.

KELECOM, Alphonse; SANTOS, Pedro Lopes dos; GOUVEA, Rita de Cássia S; DUTRA, Iedo Ramos; FEVEREIRO, Paulo Cesar Ayres. Teores elevados de Polônio-210 em plantas aquáticas da restinga de Carapebus, RJ. Química Nova, São Paulo, v.22, n.5, p.666-668, set. 1999.

KHATER, Ashraf E.M. Polonium-210 Budget in Cigarettes. Journal of Environmental Radioactivity. Volume 71(1), Pages 33-41, 2004.

LE, Mai H. Polonium 210, exposed. Journal of Medical Toxicology, Vol. 3, número 2: 83-84, 2007. 
LI, Chunsheng; SADI, Baki; WYATT, Heather; BUGDEN, Michelle; PRIEST, Nicholas; WILKINSON, Diana; KRAMER, Gary H. Metabolism of ${ }^{210}$ Po in rats: volatile ${ }^{210}$ Po in excreta. Radiation Protection Dosimetry, 140(2): 158-162. February 16, 2010.

LI, Wei Bo; GERSTMANN, Udo; GIUSSANI, Augusto; OEH, Uwe; PARETZKE, Herwig G. Internal dose assessment of 210Po using biokinetic modeling and urinary excretion measurement. Radiation and Environmental Biophysics, September, 2007.

LIN, Zhichao; WU, Zhongyu. Analysis of polonium-210 in food products and bioassay samples by isotope-dilution alpha spectrometry. Applied Radiation and Isotopes, Volume 67, Issue 5, Pages 907-912, May 2009.

MATTHEWS, K. Murray; CHANG-KYU, Kim; MARTIN, Paul. Determination of ${ }^{210}$ Po in environmental materials: A review of analytical methodology. Applied Radiation and Isotopes. Volume 65 (3) pages 267-269, March, 2007.

MARSHALL, Thomas R. The 1964 Surgeon General's Report and Americans' Beliefs about Smoking. Journal of the History of Medicine and Allied Sciences; 01; 70(2):250278, April, 2015.

MARTINS, Roberto de Andrade. Como Becquerel não descobriu a radioatividade [How Becquerel failed to discover radioactivity]. Caderno Catarinense de Ensino de Física 7: 27-45, 1990.

MELI, Maria A; DESIDERI, Donatella; ROSELLI, Carla; FEDUZZI, Laura. Assessment of 210Po in Italian diet. Food Chemistry. 155: 87-90, 2014.

MUGGLI, Monique E; EBBERT, Jon O; CHANNING, Robertson; HURT, Richard D. Waking a Sleeping Giant: The Tobacco Industry's Response to the Polonium-210 Issue. American Journal of Public Health. Vol 98, No. 9: 1643-1650, Sep, 2008.

NADEL, Simon. Correspondence: Political Lancet. The Lancet. Vol. 382: 1980, 14 december, 2013.

PERSSON, Bertil R.R; HOLM, Elis. 7Be, 210Pb, and 210Po in the surface air from the Arctic to Antarctica. Journal of Environmental Radioactivity. 1-11; Feb, 2014.

PERSSON, Bertil R.R. Polonium-210 and Lead-210 in the Terrestrial environment: A historical review. Conference Paper, 24p. October, 2009.

PLANINSEK, Petra; BENEDIK, Ljudmila; SMODIS, Borut. Comparison of various dissolution techniques for determination of Po-210 in biological samples. Applied radiation and isotopes: including data, instrumentation and methods for use in agriculture, industry and medicine; March, 2013.

RÄÄF, C. L; HOLSTEIN, H; HOLM, E; ROOS, P. Hair as an indicator of the body content of polonium in humans: preliminary results from study of five male volunteers. Journal of Environmental Radioactivity 141: 71-75, 2015. 
RADFORD, Edward P. Jr; HUNT, Vilma R. Polonium-210: A volatile radioelement in cigarretes. Science (New York, N.Y.), 143(3603), 247-9, 1964.

RADFORD, Edward P. Jr; MARTELL, E.A. Polonium-210: lead-210 ratios as an index of residence times of insoluble particles from cigarette smoke in bronchial epithelium. Inhaled Particles 4 Pt 2: 567-81, Sep. 1975.

REGO, Bryanna. The Polonium brief: a hidden history of cancer, radiation, and the tobacco industry. Isis; 100(3): 453-84, Sep, 2009.

SCOTT, Bobby R. Health Risk Evaluations for Ingestion Exposure of Humans to Polonium-210. Dose-Response. 5.2: 94-122, 2007.

SEILER, Ralph; WIEMELS, Joseph. Occurrence of ${ }^{210}$ Po and Biological Effects of LowLevel Exposure: The Need for Research. Environmental Health Perspectives 120(9), 1230-1237, 2012.

STRADY, Emilie; HARMELIN-VIVIEN, Mireille; CHIFFOLEAU, Jean François; VERON, Alain; TRONCZYNSKI, Jacek; RADAKOVITCH, Olivier. 210Po and 210Pb trophic transfer within the phytoplanktonezooplanktoneanchovy/sardine food web: a case study from the Gulf of Lion (NW Mediterranean Sea). Journal of Environmental Radioactivity 143: 141-151, 2015.

TARONI, Mattia; ZAGA, Vicenzo; BARTOLOMEI, Paolo; GATTAVECCHIA, Enrico; PACIFICI, Roberta; ZUCCARO, Piergiorgio; ESPOSITO, Mássimo. 210Pb and 210Po Concentrations in Italian Cigarretes and effective dose evaluation. Health Physics Society. 107(3): 195-199, 2014.

TIDD, Michael D. The big idea: polonium, radon and cigarettes. Journal of the Royal Society of Medicine. 101(3), 156-157, March, 2008.

TSO, T.C; HARLEY, N; ALEXANDER, L.T; Source of lead-210 and polonium-210 in tobacco. Science 153(3738):880-2; August, 1966.

YAN, Ge; CHO, Hyung-Mi; LEE, Insung; KIM, Guebuem. Significant emissions of 210Po by coal burning into the urban atmosphere of Seoul, Korea. Atmospheric Environment 54: 80-85, 2012.

WAROUX, Olivier Le Polain de; COHUET, Sandra; BISHOP, Louise; JOHNSON, Sandra; SHAW, Karen; MAGUIRE, Helen; CHARLETT, Andre; FRASER, Graham. Prevalence of and Risks for Internal Contamination among Hospital Staff Caring for a Patient Contaminated with a Fatal Dose of Polonium-210. Infection Control and Hospital Epidemiology. 32 (10):1010-5, 2011.

WINDER, Ernest L; GRAHAM, Evarts A. Tobacco Smoking as a Possible Etiologic Factor in Bronchiogenic Carcinoma: A Study of Six Hundred and Eighty-Four Proved Cases. JAMA. 143: 329-336, 1950.

ZAGÀ, Vincenzo; LYGIDAKIS, Charilaos; CHAOUACHI, Kamal; GATTAVECCHIA, Enrico. Polonium and Lung Cancer. Journal of oncology. Abril, 2011. 